American Journal of Pharmaceutical Education 2016; 80 (8) Article 134.

\title{
RESEARCH
}

\section{The Development of the Croatian Competency Framework for Pharmacists}

Iva Mucalo, PhD, MPharm, ${ }^{a}$ Maja Ortner Hadžiabdić, PhD, ${ }^{a}$ Tihana Govorčinović, MSc, MPharm, ${ }^{b}$ Martina Šarić, MPharm, ${ }^{\mathrm{c}}$ Andreia Bruno, PhD, MPharm, ${ }^{\mathrm{d}}$ Ian Bates, PhD, BPharm, ${ }^{\mathrm{d}}$

${ }^{a}$ University of Zagreb Faculty of Pharmacy and Biochemistry, Zagreb, Croatia

${ }^{\mathrm{b}}$ Croatian Chamber of Pharmacists, Zagreb, Croatia

${ }^{c}$ Community Pharmacy Kuzma and Damjan, Zadar, Croatia

${ }^{\mathrm{d}}$ FIP Collaborating Centre, University College London School of Pharmacy, London, United Kingdom

Submitted August 7, 2015; accepted November 11, 2015; published October 25, 2016.

Objective. To adjust and validate the Global Competency Framework (GbCF) to be relevant for Croatian community and hospital pharmacists.

Methods. A descriptive study was conducted in three steps: translation, consensus development, and validation by an expert panel and public consultation. Panel members were representatives from community pharmacies, hospital pharmacies, regulatory and professional bodies, academia, and industry.

Results. The adapted framework consists of 96 behavioral statements organized in four clusters: Pharmaceutical Public Health, Pharmaceutical Care, Organization and Management, and Personal and Professional Competencies. When mapped against the 100 statements listed in the GbCF, 27 matched, 39 were revised, 30 were introduced, and 24 were excluded from the original framework. Conclusions. The adaptation and validation proved that $\mathrm{GbCF}$ is adaptable to local needs, the Croatian Competency Framework that emerged from it being an example. Key amendments were made within Organization and Management and Pharmaceutical Care clusters, demonstrating that these issues can be country specific.

Keywords: competency, behavioral statement, competency framework, Global Competency Framework, Croatian Competency Framework

\section{INTRODUCTION}

Implementation of pharmacy competencies as a basic prerequisite for providing pharmaceutical care and ensuring patient treatment outcomes is increasingly supporting the development of pharmacists across sectors. ${ }^{1,2}$ Competency pertains to knowledge, skills, attitudes, and behaviors that affect an individual's role or responsibilities, relate to job performance, and are subject to improvement through training and development activities. ${ }^{3}$ Competency-based developmental frameworks are increasingly common among health professions in high-income countries and are frequently used to define standards for education and training and for career progression. ${ }^{4}$ They contain a structured assembly of behavioral competencies that can contribute to supporting practitioner development and allow for effective and sustained performance.

Corresponding Author: Iva Mucalo, University of Zagreb Faculty of Pharmacy and Biochemistry, A. Kovacica 1, 10000 Zagreb, Croatia. Tel: +3851-6394-411. Fax: +38591-48. E-mail: imucalo@pharma.hr
Culture can influence expectations of pharmaceutical services by public and regulatory bodies, and religion, traditions, history, experiences, and perceptions of medications all challenge the unified understanding of competencies in pharmacy. ${ }^{5}$ Therefore, the International Pharmacy Federation, through the Pharmacy Education Initiative (FIPEd) developed an evidenced-based Global Competency Framework ( $\mathrm{GbCF})$, a document that contains a core set of behavioral competencies that should be generally applicable for the pharmacy workforce worldwide. ${ }^{6}$ The GbCF does not imply that there should be a single global curriculum that would fit all countries, but rather that it can be taken by other countries and adapted to their own needs.

Development of a national competency framework for pharmacists in Croatia was important as it could lay the foundation for bridging the gap between traditional pharmacy education and the ever-changing demands of modern health care systems. One of the major drawbacks of the Croatian continuing eduaction (CE) model is lack of a supporting system that could assist pharmacists in identifying their learning needs and supporting their 


\section{American Journal of Pharmaceutical Education 2016; 80 (8) Article 134.}

development. Therefore, development of the Croatian Competency Framework (CCF) for pharmacists, with minimum competencies required, was a way to resolve those issues, namely assessing the differences between the established and desired levels of their performance and informing development of a competency-based curriculum to achieve the desired level of competency, as previously conducted on a "pharmaceutical care competencies" cluster. ${ }^{7}$ Furthermore, strategies for quality improvement of $\mathrm{CE}$ and continuous professional development (CPD) educational activities have been offered and could serve as a complementary supporting system for continuing education development. ${ }^{8}$

The Croatian Competency Framework could assist individuals and organizations with career planning opportunities and allow the pharmacy sector to implement useful and harmonized professional development on national level. Therefore, the aim of this research was to adapt and validate the $\mathrm{GbCF}$ to be relevant for Croatian community and hospital pharmacists.

\section{METHODS}

To develop a competency framework for community and hospital pharmacists in Croatia, a GbCF developed by the FIPEd was used. ${ }^{6}$ A descriptive study was conducted in three consecutive steps: translation, consensus development, and validation by an expert panel and public consultation. Each subsequent phase was informed by and built upon the preceding phase.

Following the standard methods of translation, the original English version of the GbCF was translated into the Croatian language by expert pharmacists familiar with terminology of the area covered by the framework and knowledgeable of English-speaking culture. Based on the Brislin translation model, the framework was then back-translated by two bilingual translators, both versions were compared for accuracy and equivalence, and any discrepancies that had occurred during the process were negotiated. ${ }^{9}$ It was further refined by pharmacists' perceptions and input from lay readers. The procedures of translation and back-translation of the framework did not reveal any changes from the original (English) version of the instrument, except the contextualization of background information relevant and applicable to the Croatian context.

During the next stage, a consensus development panel convened consisting of 10 pharmacists with expertise in community practice $(\mathrm{n}=4)$, hospital pharmacy $(n=2)$, industry $(n=1)$, regulatory affairs $(n=1)$, and academia $(n=2)$, who reviewed the list of behavioral statements to adjust the GbCF according to Croatian national needs. In adapting the $\mathrm{GbCF}$, consideration was given to the current pharmacy practice at the primary care level in Croatia to ensure its relevance for pharmacists working in a community and hospital sector. From the beginning of the data-reviewing process, an iterative process of 18 rounds of consensus development panels in the period from February to July 2014 was undertaken to evaluate and make changes to the initial competency framework. Each panel session was facilitated by a pharmacist with expertise in community pharmacy and regulatory policies, and with knowledge of the GbCF.

The group sessions lasted 180 minutes and were guided by a moderator who used an interview guide to probe participants' perceptions regarding the knowledge and skills a pharmacist must possess to meet the requirements of professional practice at a foundation level. Following the introductory lecture, panel members were introduced to the GbCF's development and validation process, protocol, and structure of the panel sessions. Objectives that needed to be addressed during the adaptation process were presented and included the following considerations: whether the language was clear and understandable; whether the clustering was correct (ie, whether the labels assigned to each cluster made sense and if not, whether they could be rephrased or replaced; whether there was any competency and/or behavior missing considering the Croatian setting; and whether there was any competency and/or behavior not applicable to the Croatian setting.

Panel members were then asked to reach consensus on each behavioral statement bearing in mind its usefulness and applicability, and recommend amendments and inclusions if necessary. Finally, panel members were asked to categorize the competencies into clusters. The panel sessions were audio recorded. Within a week after a session, member checking was performed by e-mailing a summary of the discussion to the participants and asking them to confirm its accuracy.

The draft framework that emerged from the consensus development group was evaluated for content validity by pharmacists with experience in community pharmacy $(\mathrm{n}=2)$, hospital pharmacy $(\mathrm{n}=1)$, professional body $(n=1)$, regulatory body $(n=1)$, and academia $(n=2)$. Individuals who participated in the expert panel group did not attend the consensus development panels.

An iterative process of five rounds of expert panel groups in the period from July to September 2014 was undertaken to evaluate and make changes to the competency framework yielded by the previous group. At the start of each session, the procedure was explained briefly, and theoretical saturation was considered to have been reached when a new session yielded no new information. 


\section{American Journal of Pharmaceutical Education 2016; 80 (8) Article 134.}

Each revised competency cluster was reviewed to ensure consistency of terminology and overall content, and to reevaluate its applicability and usefulness for general level primary care. Before the first meeting, the panel members were invited to rank the relevance (on a 5 -point Likert scale where $1=$ not relevant and $5=$ very relevant) and wording of the behaviors and competencies, after which they received written feedback comprising the means and standard deviations of the relevance scores and a summary of the textual comments. They were also asked to edit or reword each statement they felt needed revision and to provide additional information as appropriate. In the absence of an existing standard, a strict definition of consensus was used: a competency had to be rated as relevant (4) or very relevant (5) by at least $80 \%$ of panel members. ${ }^{10}$ Minor revisions were made to the wording in response to comments from panel members.

The amended framework underwent a final review (fifth session) by an expert panel before distribution to members of the Croatian Pharmacy Chamber, all registered pharmacists, for final critique. The final competency framework resulting from this process was made available for a public consultation with the aim of collecting comments and suggestions from members of the Croatian Pharmacy Chamber before drafting the final document. The process consisted of three related forms of interaction with interested members of the pharmacy public: notification, consultation, a 2-way flow of information, using an online survey-tool via the chamber's website, and participation, an active meeting of interest groups, held at the University of Zagreb Faculty of Pharmacy and Biochemistry to increase the sense of ownership of, or commitment to, the framework beyond what is likely to be achieved via a purely consultative approach.

All participants in the consensus development groups and the expert panel groups gave informed consent in response to a letter that explicitly stated that participation was voluntary and that gave assurance of full confidentiality, pursuant to the Croatian "regulations on the duty of protection of professional and official confidential information" (IRB number: 330-02-114-10).

\section{RESULTS}

The purpose of this study was to adapt and validate a framework of pharmacy competencies relevant for community and hospital setting by examining whether pharmacy experts could reach consensus. In this sample of 17 pharmacists [gender: all female; mean age 42.15 (18.2) years], overall mean duration of work experience in the pharmacy setting was 18.15 (9.2) years.

An initial draft of the CCF for pharmacists was developed from the source framework $\mathrm{GbCF}$ and methods for establishing and developing consensus followed by public consultation. One hundred behavioral statements, grouped in four competency clusters, were used for the review from the GbCF. The mapping of the behavioral statements from the source document and expert panel confirmed the importance of these four existing competency clusters, although amendments were necessary (Table 1). When CCF was mapped against the 100 behavioral statements listed in the GbCF, 27 could be found in the adapted framework, 39 were revised, 30 were newly introduced, and 24 from the original framework were excluded. A detailed overview of amendments can be found in Table 2. The scope of the CCF encompasses foundation-level or early-years practice and represents national consensus on the capability competencies of the outcomes of registration levels of initial career education and training.

The panel members of the consensus development group agreed about changing some competency headings, behavior wording and descriptors for clarification purposes, amalgamation of two competencies into one as to display the process of working (such as procurement and the ancillary supply chain management) as interconnected, transfer of a competency from one cluster to another, change of behavioral statement order within the competency to present the sequence of activities in a more logical manner, and the change of competency order within clusters. Some of the most significant amendments

Table 1. A Comparison between the Global Competency Framework (GbCF) and Croatian Competency Framework (CCF) in Number of Competencies and Behavioral Statements Across Four Clusters

\begin{tabular}{lccrc}
\hline & \multicolumn{2}{c}{ No. of Competencies } & \multicolumn{2}{c}{ No. of Behavioral Statements } \\
\cline { 2 - 5 } Competency Cluster & GbCF & CCF & GbCF & CCF \\
\hline Pharmaceutical Public Health & 2 & 1 & 3 \\
Pharmaceutical Care & 6 & 5 & 25 & 32 \\
Organisztion and Management & 6 & 5 & 39 & 36 \\
Personal and Professional & 6 & 6 & 100 \\
Total & 20 & 17 & 96 \\
\hline
\end{tabular}


American Journal of Pharmaceutical Education 2016; 80 (8) Article 134.

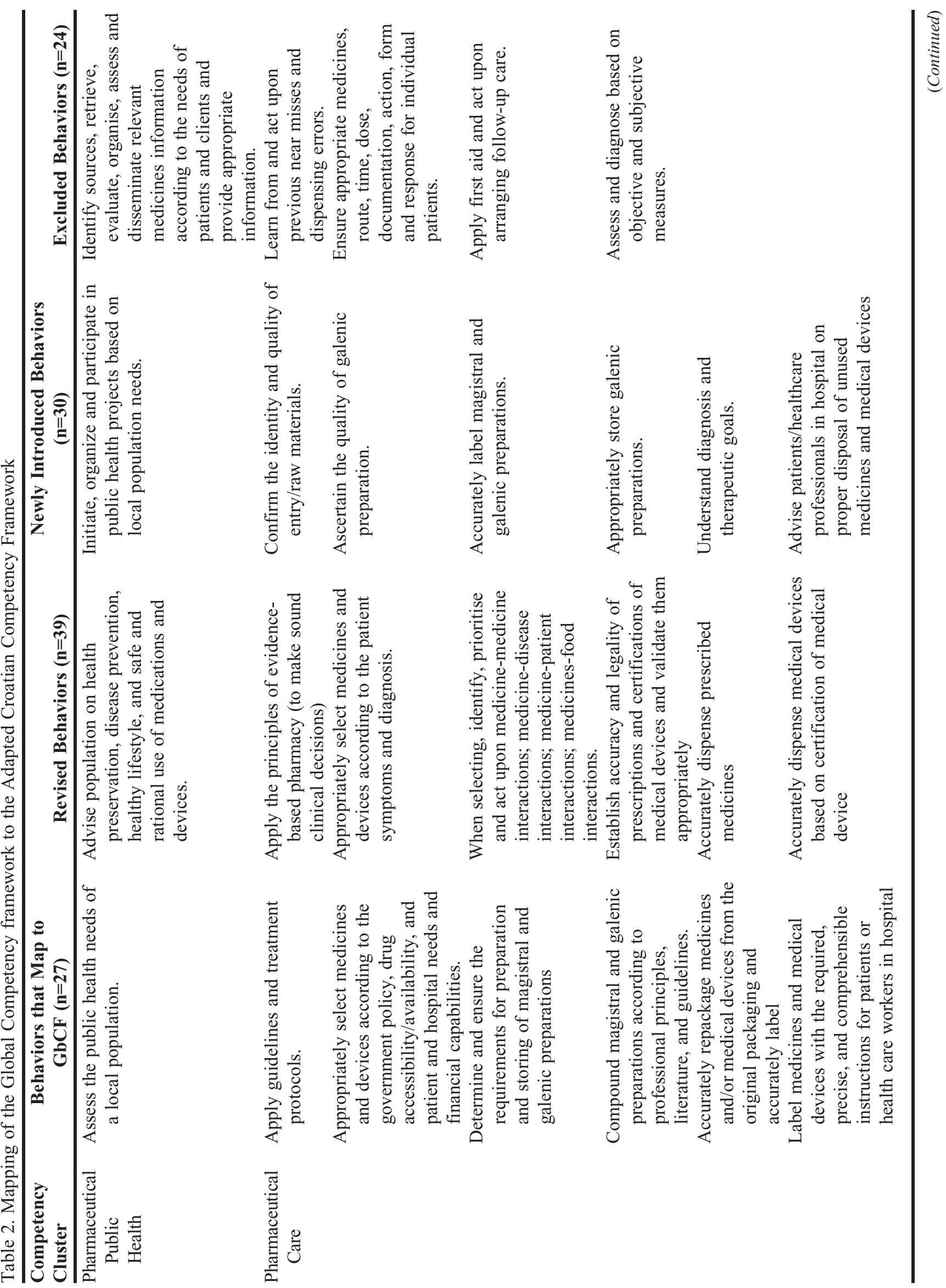


American Journal of Pharmaceutical Education 2016; 80 (8) Article 134.

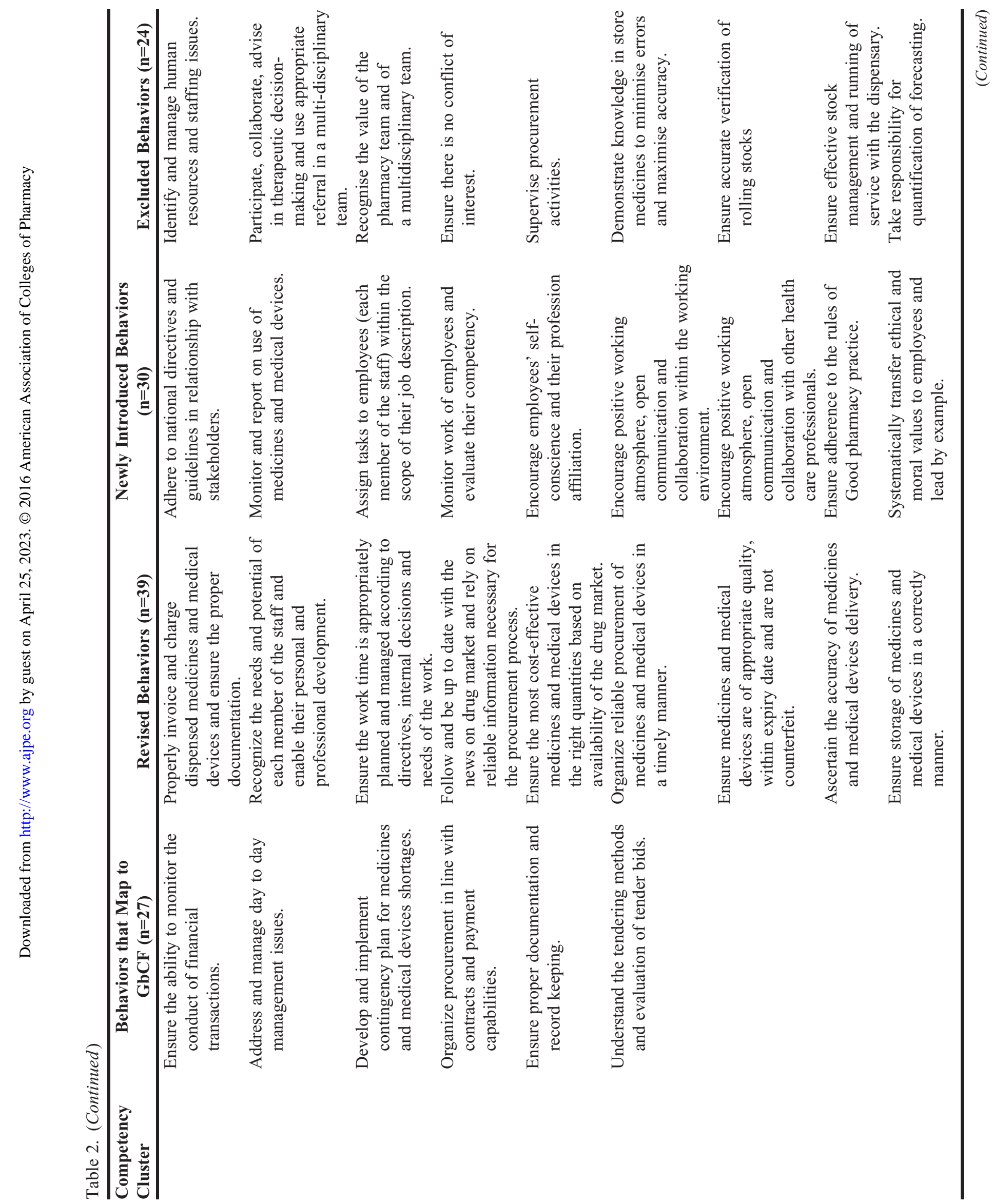


American Journal of Pharmaceutical Education 2016; 80 (8) Article 134.

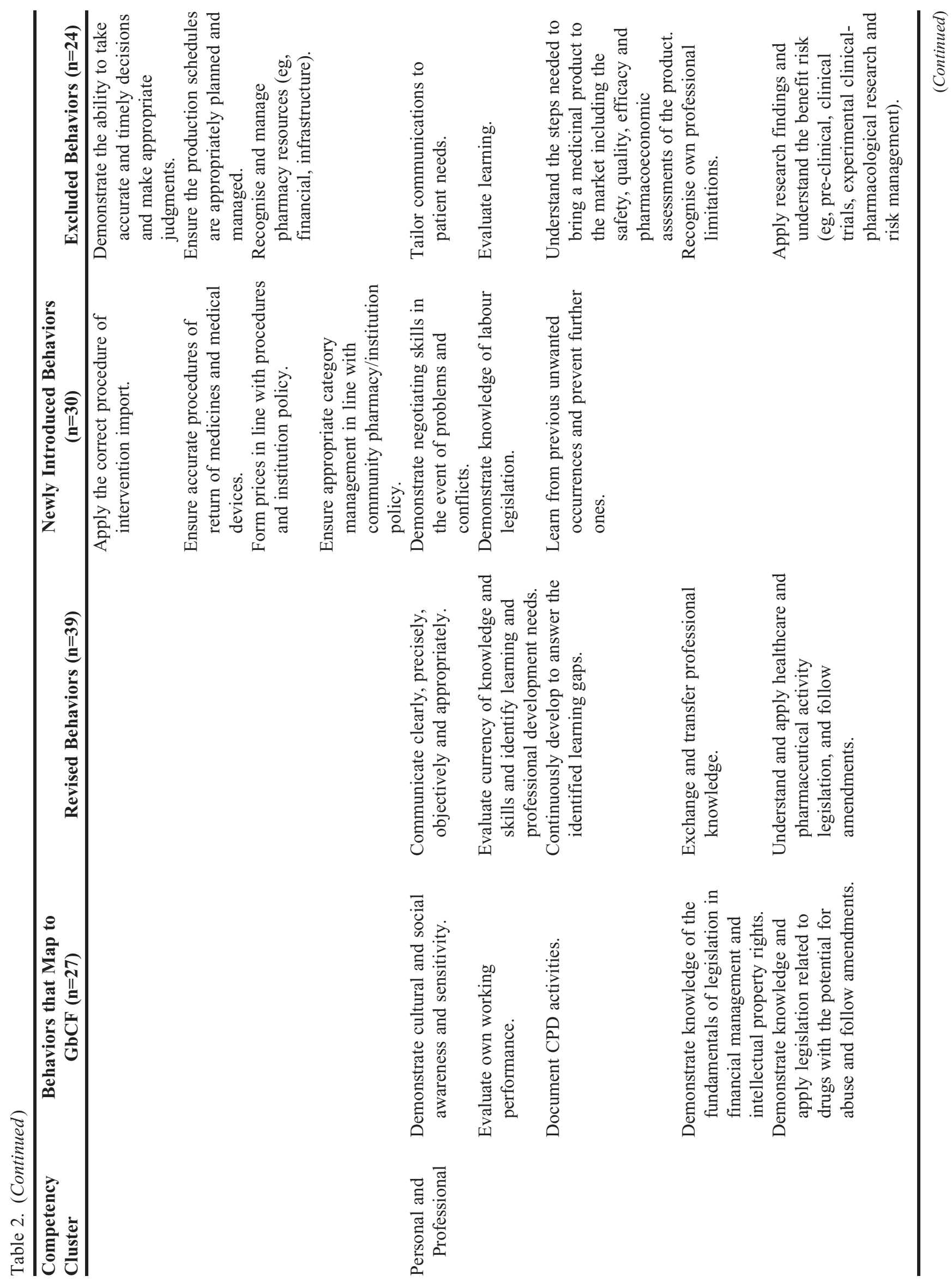


American Journal of Pharmaceutical Education 2016; 80 (8) Article 134.

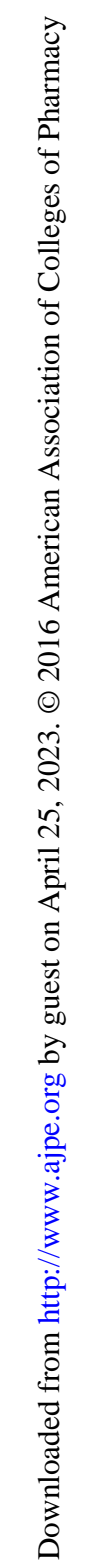

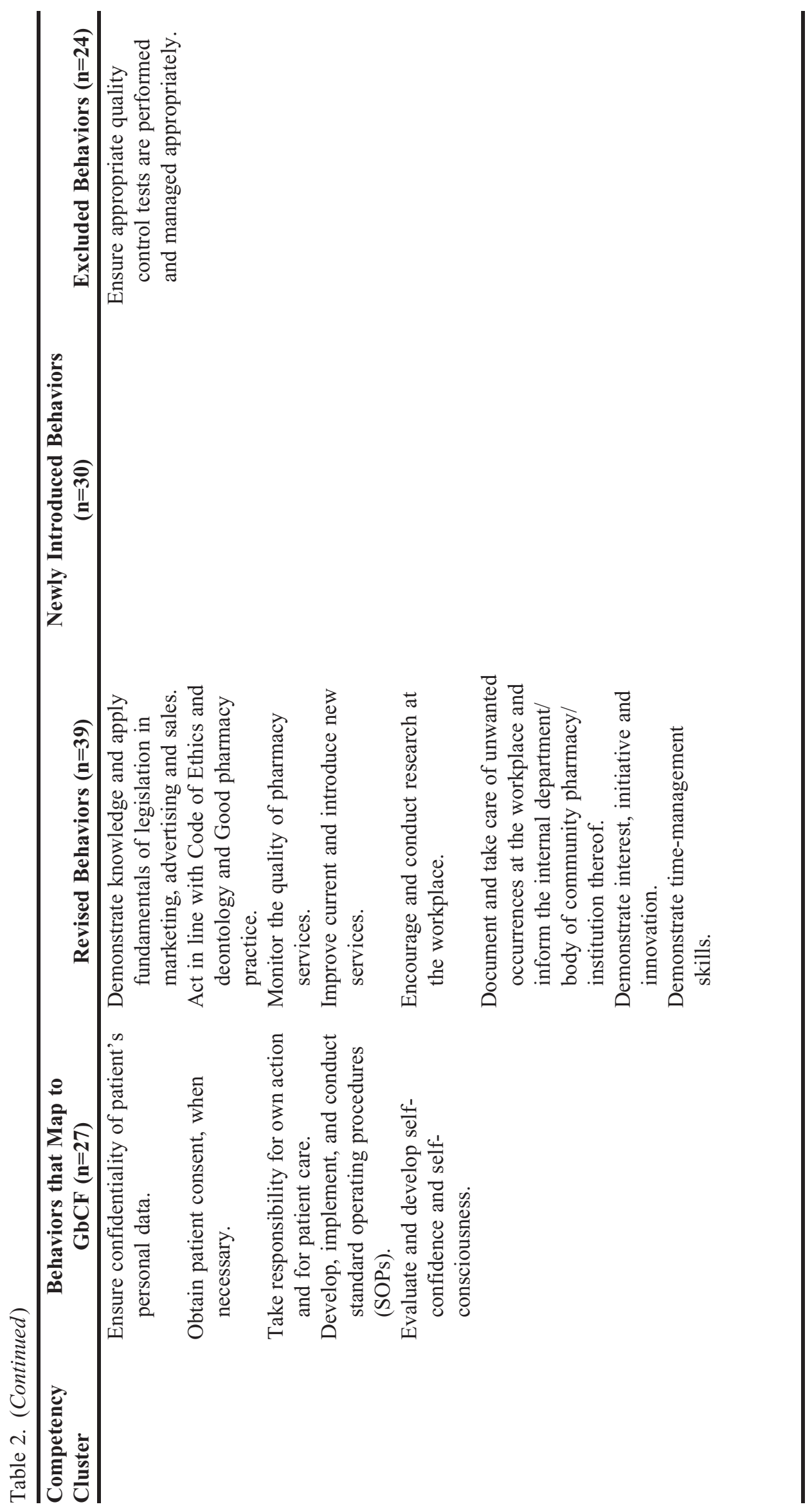




\section{American Journal of Pharmaceutical Education 2016; 80 (8) Article 134.}

to the CCF included addition of new competencies (eg, "Assurance of safe medicines use" in the Pharmaceutical Care cluster, "Organization" in the Organization and Management cluster) and exclusion of three competencies ("Medicines information and advice" from the Pharmaceutical Public Health cluster, "Medicines" and "Monitor medicines therapy" from the Pharmaceutical Care competences cluster).

Further amendments encompassed exclusion of competencies that were too broad or unspecific, immeasurable (eg, "Recognize the value of the pharmacy team and of a multidisciplinary team"), not yet present in Croatian pharmacy practice (eg, "Ensure appropriate quality control tests are performed and managed appropriately in Quality Assurance and Research in the workplace competency), or too advanced for foundation level framework (eg, Apply research findings and understand the benefit risk (eg, preclinical, clinical trials, experimental clinicalpharmacological research and risk management).

Several statements of similar content (eg, "Identify if expertise needed outside the scope of knowledge" and "Identify learning needs") were replaced for one behavioral statement. Additional modifications included clarifying the "Compound medicines" competency by including additional behaviors regarding extemporaneous preparations compounding and storage; combining "Procurement" and "Supply-chain management" competencies and adding behaviors regarding procurement and supply chain management; adding behaviors relating to "Patient consultations" competency in the Pharmaceutical Care cluster; and adding behavior statements regarding human resources and workplace management in the Organisation and Management cluster.

After the adaptation process, the mean relevance scores of each behavioral statement and some minor adjustments of the competencies (eg, wording) were reached by the expert panel members. A summary of relevancy mean scores and standard deviations for all behavioral statements is detailed in Table 3. Ninety-four percent $(n=90)$ of behavioral statements were rated as relevant by more than $80 \%$ of respondents. During the validation process, consensus was reached on keeping the remaining six statements as they were considered relevant for Croatian practice. The "Encourage and conduct research at the workplace" [3.9 (0.69), N=7], "Understand the tendering methods and evaluation of tender bids" [3.7 (1.11), $\mathrm{N}=7$ ], and "Monitor and report on use of medicines and medical devices [3.9 (0.69), $\mathrm{N}=7$ ], showed the least relevance. This may reflect the absence of workplace research culture. Furthermore, tendering is a method applicable for hospital pharmacy and a small proportion of community pharmacies supplying prison and nursing home facilities and was thus recognized as important by a lower proportion of pharmacists present in the expert panel. Moreover, monitoring and reporting on the use of medications and medical devices is usually carried out by a community or hospital pharmacy manager and so was not recognized by the remaining pharmacy personnel as highly important. Nevertheless, these statements followed the validation process considered important for foundation level practice, and consensus was reached on retaining them.

The expert panel agreed to the majority of amendments proposed by the consensus development panel and further refined the framework in terms of uniformity of language, wording, and some minor amendments not affecting the content itself, bearing in mind the perspective and the context of the Croatian pharmacy profession.

After public consultation with all registered pharmacists $(n=3431)$, only 26 responses were received, all of which were included in the final draft. Those comments addressed the issues related to procurement and supply chain management and patient consultation competencies clarification, distinctions between hospital and community pharmacy dispensing and adverse drug reactions reporting, and organizing and providing competencyinformed education for registered pharmacists. The biggest fear expressed by pharmacists was not being up to date with knowledge and skills to satisfy the competencies in the competency framework. All pharmacists found the framework clear and easy to understand and stated that the framework reflected the core competencies of a community/hospital pharmacist. Many useful comments and suggestions were also received from pharmacists on how the framework could be improved, and pharmacists provided additional behaviors useful to the framework. The framework could, therefore, be considered the competency standards set by both the regulatory body and the profession itself.

Upon consultation with the profession, the framework was updated and finalized to reflect the comments and suggestions received during the consultation process. The final framework consisted of 96 behavioral statements, grouped in 17 competencies under four main competency domains: Pharmaceutical Public Health, Pharmaceutical Care, Organisation and Management, and Personal and Professional. A detailed overview of the framework is given in Table 3.

\section{DISCUSSION}

This is the first comprehensive Croatian Competency Framework eligible for supporting pharmacists working in community and hospital sector in Croatia. 
American Journal of Pharmaceutical Education 2016; 80 (8) Article 134.

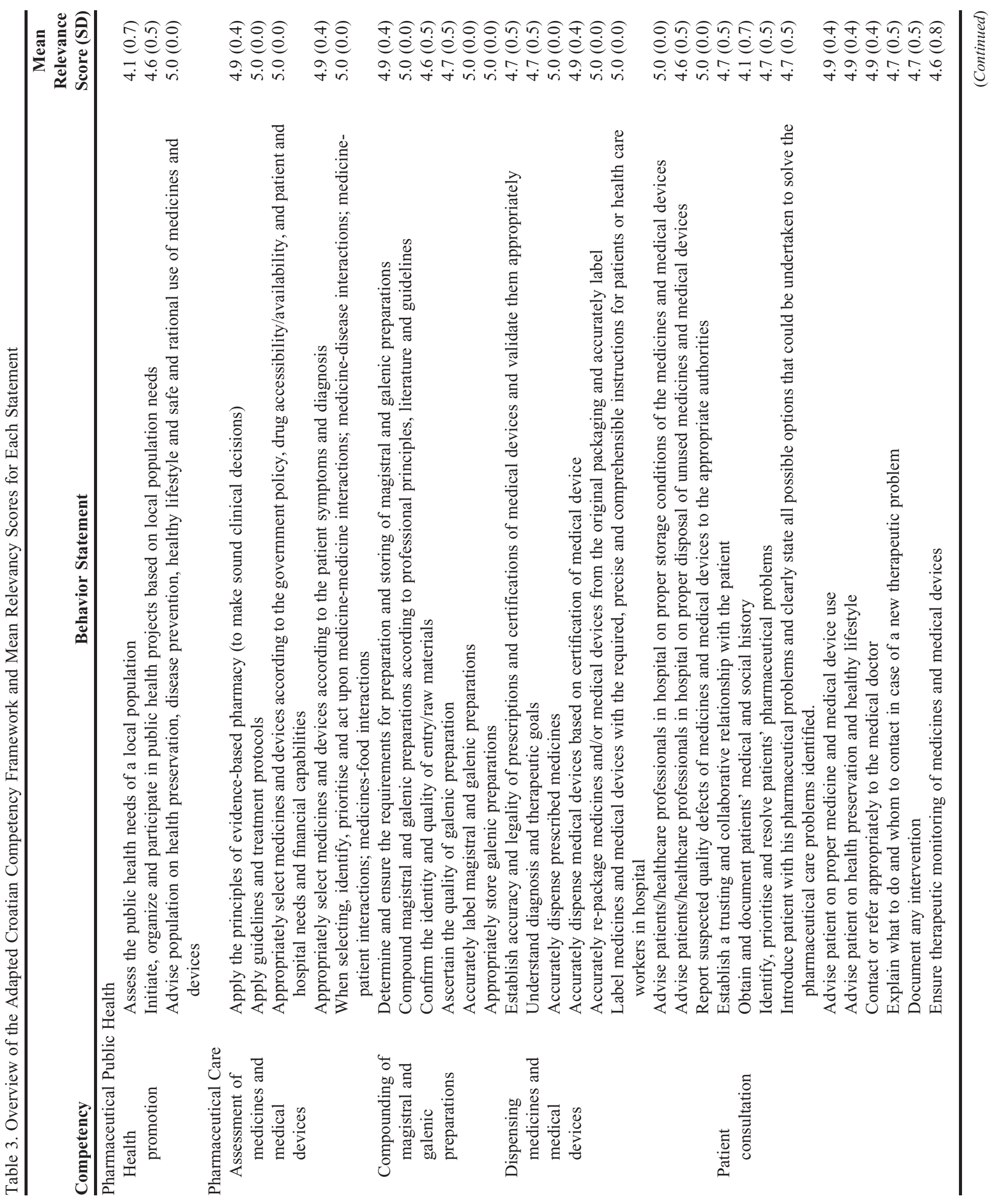


American Journal of Pharmaceutical Education 2016; 80 (8) Article 134.

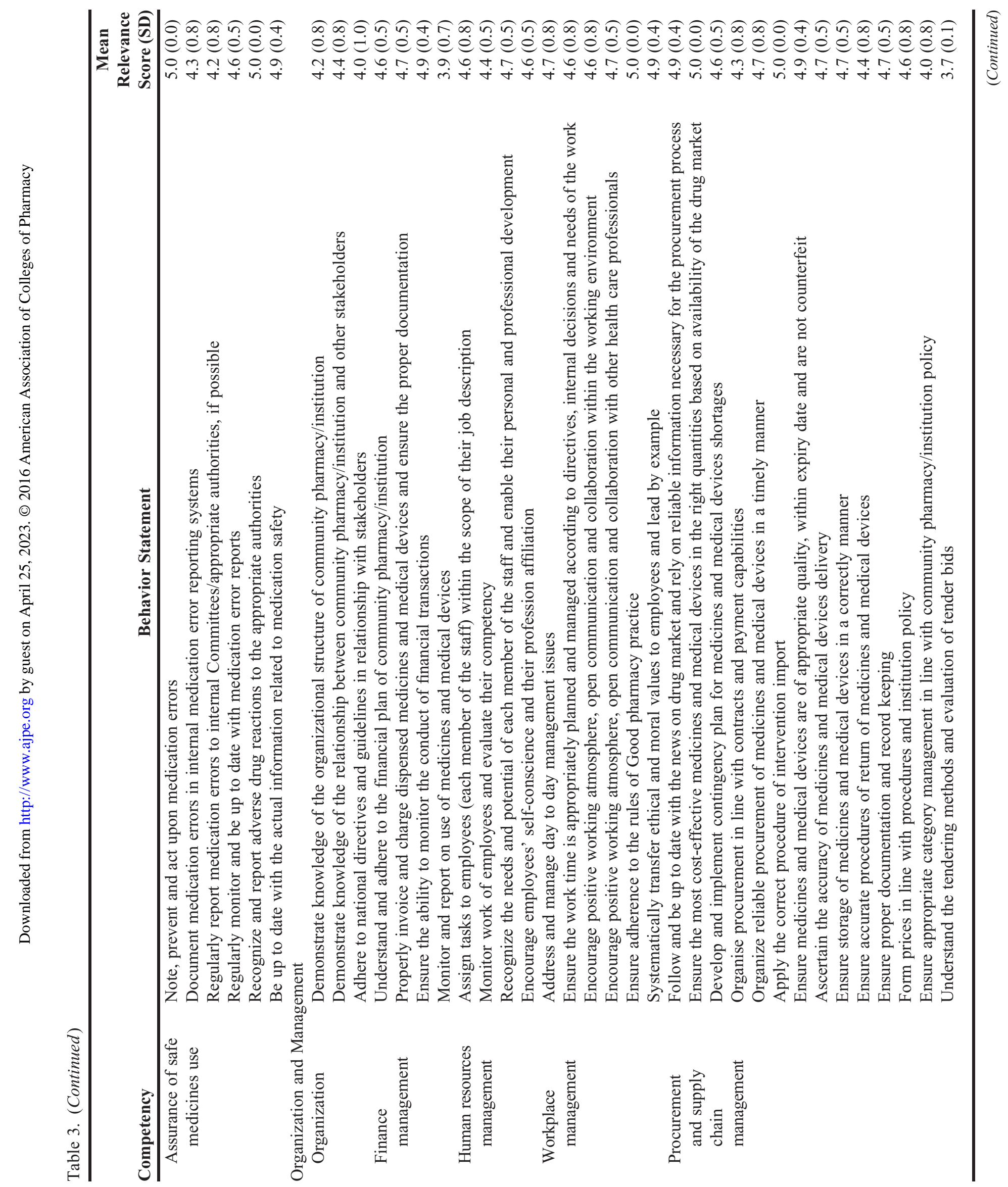


American Journal of Pharmaceutical Education 2016; 80 (8) Article 134.

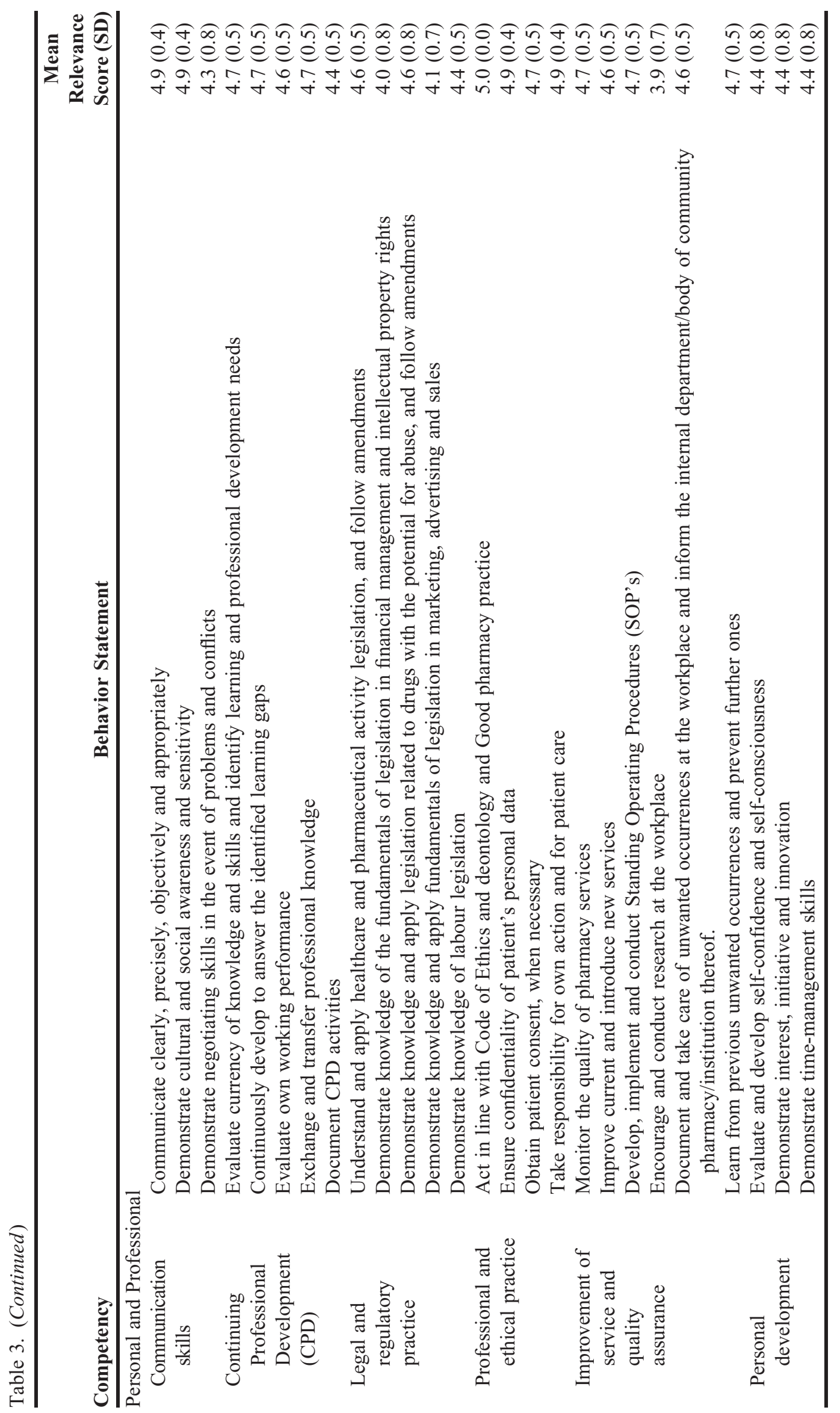




\section{American Journal of Pharmaceutical Education 2016; 80 (8) Article 134.}

The adapted framework reflects the current status of pharmacy practice in Croatia and lays foundation for the new pharmacy contract with Croatian Health Insurance Fund regarding the provision of advanced services. Moreover, Croatia is among the first non-English speaking countries in Southeastern Europe to have used, properly adjusted, and validated the global framework, adapting it to its national needs. The assessment of Croatian community pharmacists' patient care competencies using the General Level Framework was previously investigated and provided a foundation for the enforcement of the current study. ${ }^{11}$ Other countries from the region that are developing competency frameworks include Serbia, ${ }^{12}$ Slovenia, ${ }^{13}$ and Monte Negro, whereas Lithuania and Turkey have recently started with implementation. The CCF employed methodology similar to the one used by Ireland, the first country to have adapted the GbCF, with the exception of translation and cross-cultural adaptation. ${ }^{14}$ Both frameworks, although similar in structure, yielded two dissimilar documents.

Croatia has a different pharmacy practice tradition compared to Anglo-Saxon countries that already use competency frameworks in various health care sectors and are consequently more accustomed to it. ${ }^{15-22}$ Concepts of pharmaceutical care and clinical pharmacy, namely patient-centred practices are underdeveloped and lack inclusion in the nation's health care system. In addition, competencies per se are a part of educational paradigm which is not yet fully implemented in our educational system and much effort was invested to disseminate information regarding its relevance and educate pharmacy public on the matter. The process is not completed and time is needed for the concept to be comprehended and accepted by Croatian pharmacists.

Despite the fact that the adaptation of a culturally different GbCF presented quite a challenge, we strongly believe that the process could serve as an example to other European countries with similar pharmacy practicerelated issues that have not yet developed their own country-specific framework. We found that although there were barriers in the process of creating the national competency framework, they could be overcome by taking into account standard methods of translation and cross-cultural adaptation. ${ }^{23-26}$ Furthermore, we confirmed that the methodology used in this process could yield a country-specific framework that we recommend to other countries.

When comparing GbCF and CCF, most differences are in the Pharmaceutical Care and Organization and Management competency clusters. Although considerable congruence was noticed between the original and adapted document in Pharmaceutical Care, additional be- haviors were needed. The framework was primarily modified within the Patient Consultation competency, which grew to 10 from the original six statements. For years, Pharmaceutical Care remained unrecognized and unpracticed along with its key patient-centered skill, patient consultation, and pharmacists were merely advice-givers. In the light of changes and acknowledged necessity for pharmaceutical care development, as well as the importance of the consultation skills as a 2-way process, authors considered it essential to disassemble this composite process to consecutive steps and add more behaviors as to familiarize pharmacists with the sequence of activities within the Patient Consultation cluster.

The most amended cluster was Organization and Management, where approximately half of the competencies were replaced and the other half were revised. This highlights the importance of adapting the generic global competency framework in organizational and managerial issues to the local and national circumstances, as these can be country-specific. Although pharmaceutical care is a universal practice not necessarily related to culture, tradition, and history, it differs from country to country depending on the level of its development.

As for statements that represent the future pharmacists' roles but are not yet incorporated in the health care system (eg, reporting document medication errors in internal medication error reporting systems; reporting medication errors to internal committees/appropriate authorities), most were retained with an aim of raising awareness about them, especially when using the framework in the undergraduate context.

Following public consultation, a low response rate was obtained, as expected, reflecting pharmacists' poor engagement, initiative, and interest in competencies and their implementation. Unfortunately, this is a result of the lack of understanding of the concept and importance of competencies by the pharmacy profession. However, an unexpectedly high proportion of pharmacists $(\mathrm{N}=98)$ answered the invitation and participated in the closing event, reporting that they wanted to contribute to the final draft. This demonstrated a slow, but existent, shift in pharmacists' perceptions and assisted in forming a new climate in Croatian community and hospital setting.

The adapted framework was created following an evidence-based approach and was validated by an expert and individual pharmacists' opinion. The pharmacists working across various pharmacy sectors received an official invitation for participation in the project from the Croatian Pharmacy Chamber, and had subsequently been recruited. A pragmatic approach to recruitment was adopted because of the high level of commitment required of participants over a long period of time. Within the study 


\section{American Journal of Pharmaceutical Education 2016; 80 (8) Article 134.}

period, 23 session rounds (workshops with an average duration of 180 minutes) were organized, suggesting a high level of enthusiasm and support. All participating pharmacists continued their involvement in the project throughout the one-year period as they were eager to contribute to the development of the framework, which confirmed the need for a competency framework.

The adapted framework will inform the curriculum development, serve as a basis for pharmacists' training in community and hospital setting, provide a foundation for a new CPD model, and offer support for workforce development. The CCF will be tested among student populations during their 6-month preregistration period and among practicing pharmacists, during which their clinical activities will prospectively be recorded at several time points. Two methods of students' competency assessment will be employed: self-assessment by students at two time-points (following six weeks and six months) and vertical assessment conducted on behalf of students' mentors/preceptors at the end of the preregistration training to evaluate student performance. The evaluation of the CCF will demonstrate whether the adapted framework sustains pharmacists' development and is efficient at developing the competence of students in community and hospital pharmacy towards a registered pharmacist. Following the initial implementation of the framework, authors intend to continue refining and validating the framework in regard to ambiguities, inapplicability, or lack of clarity of certain behaviors.

Further research is needed to determine whether the methodology applied in our study can serve as a model for adapting and developing other countries' frameworks, especially in the countries with similar pharmacy practice policy, and whether the CCF can role-model and inform that development.

We instigated this research as the profession needs a systematic and standardized platform to demonstrate to other health care professionals, patients, and pharmacists the role we play in the safe and responsible pharmaceutical care provision. By actively incorporating quality improvement through competency-based framework, pharmacists can bring about systematic changes about how patient care is delivered.

The current study was limited in several ways. First, the selection of the expert panel members and the question of sample representativeness was raised, especially when considering that all participants were female. However, consensus panel methodology typically utilizes nonrandom sampling because of the need for expert identification; in fact, literature consistently supports the use of selected panelists. ${ }^{27,28}$ Additionally, based on the data from Croatian Chamber of Pharmacists, gender distribution is predominantly female (93\%). Further issues included: panel members not being a representative population, which makes the findings difficult to generalize; participants having subjective views that may have fluctuated over time; and group dynamics potentially restricting some panel members from voicing their opinions. To minimize those limitations, the facilitator attempted to enable a balanced discursive contribution among panel members. Another limitation was that the CCF is specifically tailored to the country's current pharmacy practice situation.

Considering the changing environment of pharmacy practice, we recommend an ongoing process of competency framework evaluation and validation to ensure its sustained value in the future. Finally, an issue on equal competency comprehension by all pharmacists was raised. Indeed, without the description of each behavioral statement, one could not be certain that all pharmacists comprehended the listed competencies and the pertaining behaviors in the same manner, even with the provided explanation in a form of a handbook.

\section{CONCLUSION}

This adaptation and validation demonstrated that a global framework can be adaptable to local needs, as the Croatian Competency Framework emerged from the $\mathrm{GbCF}$, and can be used for creating a country-specific mapping tool. Key amendments were made in the Organization and Management and Pharmaceutical Care competencies clusters, demonstrating that these issues can be country-specific.

\section{ACKNOWLEDGMENTS}

The authors wish to thank panel members and colleagues who provided assistance with the research.

\section{REFERENCES}

1. Hill LH, Delafuente JC, Sicat BL, Kirkwood CK. Development of a competency-based assessment process for advanced pharmacy practice experiences. Am J Pharm Educ. 2006;70(1):Article 1. 2. Mills E, Bates I, Farmer D, Davies G, Webb DG. The General Level Framework - use in primary care and community pharmacy to support professional development. Int J Pharm Pract. 2008;16(5): 325-331.

3. Lucia AD, Lepsinger R. The Art and Science of Competency Models: Pinpointing Critical Success Factors in Organizations. Hoboken, NJ: Jossey-Bass; 1999.

4. Hendry C, Lauder W, Roxburgh M. The dissemination and uptake of competency frameworks. J Res Nursing. 2007;12(6):689-700. 5. Bruno A, Bates I, Brock T, Anderson C. Towards a global competency framework. Am J Pharm Educ. 2010;74(3):Article 56. 6. Pharmacy Education Taskforce. A Global Competency Framework. 2012; https://www.fip.org/files/fip/PharmacyEducation/ GbCF_v1.pdf. Accessed June 5, 2015. 


\section{American Journal of Pharmaceutical Education 2016; 80 (8) Article 134.}

7. Meštrović A., Staničić Ž., Ortner Hadžiabdić M., et al. Individualized education and competency development of Croatian community pharmacists using the General Level Framework. Am J Pharm Educ. 2012;76(2):Article 23.

8. Meštrović A, Rouse MJ. Pillars and foundations of quality for continuing education in pharmacy. Am J Pharm Educ. 2015;79(3): Article 45.

9. Brislin RW. Back-translation for cross-cultural research. J Cross Cult Psychol. 1970;1(3):185-216.

10. Holey EA, Feeley JL, Dixon J, Whittaker VJ. An exploration of the use of simple statistics to measure consensus and stability in Delphi studies. BMC Med Res Methodol. 2007;7(1):52. 11. Meštrović A, Staničić Z, Hadžiabdić MO, et al. Evaluation of Croatian community pharmacists' patient care competencies using the General Level Framework. Am J Pharm Educ. 2011;75(2):Article 36. 12. The Pharmaceutical Chamber of Serbia. National framework for pharmacists' competency assessment. 2014; http://www.farmkom.rs/ images/stories/news/vesti/nacionalni-okvir-za-procenu-kompetencijafarmaceuta-draft.pdf. Accessed October 10, 2015.

13. Slovene Chamber of Pharmacy. Competency catalog for pharmacists. 2012, Slovene Chamber of Pharmacy, Ljubljana, Slovenia. 14. The Pharmaceutical Society of Ireland. Core Competency Framework for Pharmacists. 2013. http://www.thepsi.ie/gns/ pharmacy-practice/core-competency-framework.aspx. Accessed on 1 October 2015.

15. Competency Development and Evaluation Group. General Level Framework, a Framework for Pharmacist Development in General Pharmacy Practice. 2007; http://www.codeg.org/fileadmin/codeg/ pdf/glf/GLF_October_2007_Edition.pdf. Accessed July 17, 2015. 16. Global Conference Proceedings. The Basel Statements on the future of hospital pharmacy. Am J Health-Syst Pharm. 2009; 66 (Suppl 3):S61-S66.

17. National Association of Pharmacy Regulatory Authorities. Model Standards of Practice for Canadian Pharmacists. 2003; http:// napra.ca/Content_Files/Files/Model_Standards_of_Prac_for_Cdn_ Pharm_March09_Final_b.pdf. Accessed July 17, 2015.

18. Pharmaceutical Society of Australia. National Competency Standards Framework for Pharmacists in Australia. 2010; https:// www.psa.org.au/downloads/standards/competency-standardscomplete.pdf. Accessed July 15, 2015.
19. Pharmacy Council of New Zealand. Competency Standards for the Pharmacy Profession. 2006; http://www.pharmacycouncil.org.nz/ cms_show_download.php?id=501. Accessed July 17, 2015. 20. The Council on Credentialing in Pharmacy. Scope of Contemporary Pharmacy Practice: Roles, Responsibilities, and Functions of Pharmacists and Pharmacy Technicians. 2009. http:// www.jmcp.org/doi/pdf/10.18553/jmcp.2010.16.7.507

21. World Health Organization. A model quality assurance system for procurement agencies (recommendations for quality assurance systems focusing on prequalification of products and manufacturers, purchasing, storage and distribution of pharmaceutical products). 2007. http://apps.who.int/prequal/ info_general/documents/TRS937/WHO_TRS_937_annex6_eng. pdf. Accessed July 18, 2015.

22. Essential Drugs and Medicines Policy, Interagency Pharmaceutical Coordination Group. Operational principles for good pharmaceutical procurement. 1999. http://www.who.int/3by5/en/who-edm-par-99-5. pdf. Accessed July 18, 2015.

23. Sousa VD, Rojjanasrirat W. Translation, adaptation and validation of instruments or scales for use in cross-cultural health care research: a clear and user-friendly guideline. J Eval Clin Pract. 2011;17(2):268-274.

24. Brislin RW. The wording and translation of research instruments. In: Lonner WJ, Berry JW, eds. Field Methods in Cross-Cultural Research. Beverly Hills, CA: Sage Publications; 1986:137-164. 25. Chapman DW, Carter JF. Translation procedures for the cross cultural use of measurement instruments. Eduzc Eval Policy Anal. 1979;1(3):71-76.

26. Wild D, Grove A, Martin M, et al. Principles of good practice for the translation and cultural adaptation processs for patientreported outcomes (PRO) measures: report of the ISPOR task force for translation and cultural adaptation. Value Health. 2005;8(2): 94-104.

27. Ludwig B. Predicting the future: have you considered using the Delphi Methodology? J Ext. 1997;35(5):1-4.

28. Shelton K, Adair Chreghan K. Demystifing the Delphi method. In: Information Resources Management Association, ed. Research Methods: Concepts, Methodologies, Tools, and Applications. Hershey, PA: Information Science Reference; 2015:84-105. 